ment compléter le corps de l'ouvrage: on y trouve l'évolution de la densité médicale, le nombre et l'origine des étudiants en médecine, la liste des autorités politiques et universitaires et le nom des professeurs ayant enseigné ou enseignant actuellement à la Faculté.

L'ensemble forme un ouvrage de "peu de mots et de beaucoup d'images» (l'auteur dixit) où le lecteur trouvera une foule de renseignements sur le corps professoral lausannois et sur les lieux où ces médecins exercèrent leur activité. Il a les qualités et les défauts d'un ouvrage commémoratif - avec sa glorification des sommités médicales locales - et on peut douter qu'à une époque où la médecine scientifique suscite autant de craintes que de fascination, il atteigne entièrement le but recherché, à savoir «définir un héritage commun, reserrer les liens du personnel de santé, comme entre Faculté et grand public». Mais ce livre constitue un très bel album de famille de la Faculté lausannoise et il sera consulté avec profit par un lectorat cultivé, intéressé à l'histoire locale, désireux de connaître les antécédents de la Faculté de médecine et du Centre hospitalier universitaire et d'apprendre comment ces institutions ont conquis la place qu'elles occupent aujourd'hui dans la capitale vaudoise.

Jean Jacques Dreifuss

Jacques Gasser, Photographie et médecine 1840-1880. Avec la collaboration de Stanley B.Burns. - Lausanne, Institut universitaire d'histoire de la médecine et de la santé publique, 1991. 47 S.Ill. SFr.35.-. ISBN 2-9700002-0-2.

Ce catalogue illustré de l'exposition qui s'est tenue au Musée suisse de l'appareil photographique de Vevey au printemps 1991 débute par une introduction du Dr Gasser, qui relate l'histoire des débuts de la photographie médicale. Cette introduction est suivie d'un article de Stanley B.Burns, ophtalmologue new-yorkais, connu par ses contributions à l'histoire de la photographie médicale. Il est, en particulier, le créateur de The Burns Archive, une collection photographique riche de plus de 100000 documents, dont 2000 images prises entre 1839 et 1920 . Intitulé «Les incunables photographiques de la médecine», cet article traite des premiers daguerréotypes et des autres images photographiques «à usage unique» de cette époque. Mais l'intérêt de cet album est encore fait d'une quinzaine de reproductions, soigneusement choisies, et du catalogue proprement dit. Ce dernier contient une notice brève mais complète, et parfois illustrée, de chacun des 67 documents présentés à l'exposition. Défilent ainsi tour à tour, après «Les 
incunables», toute une série de photographies réunies sous les thèmes «La chirurgie», «Le portrait psychiatrique», «Duchêne et l'expression des passions», «Les débuts de la neurologie», «La dermatologie» et, enfin, «Curiosités», ce dernier chapitre illustrant de nombreux cas de tératologie. Il se termine par une brève chronologie, un glossaire et une bibliographie indicative.

L'exposition ayant fermé ses portes le 9 juin 1991, ce catalogue constitue un document qui en perpétuera le souvenir, et une très intéressante contribution iconographique à l'histoire de la médecine.

Roger Mayer

Du 27 février au 30 avril 1992 l'exposition «Photographie et Médecine 1840$1880 »$ sest présentée au Musée d'histoire de la médecine à Zurich :

Medizinhistorisches Museum der Universität Zürich, Rämistrasse 69, 8001 Zürich.

Heures d'ouverture: du mardi au vendredi 13.00-18.00, samedi et dimanche $11.00-17.00$.

\section{Adresses of reviewers}

Prof. Dr.med. Jean Jacques Dreifuss, Département de Physiologie, Centre médical universitaire, 1, rue Michel Servet, 1211 Genève 4

PD Dr. med. Roger Mayer, 9, rue Michel-Chauvet, 1208 Genève

Prof. Dr. sc.nat. Rudolf Trümpy, Allmendboden 19, 8700 Küsnacht 